\title{
Trayectoria y balance en la historiografía peruana: 90 años de la Academia Nacional de la Historia (1905-1995)
}

Teodoro Hampe Martínez

Pontificia Universidad Católica del Perú

Con el objeto de analizar el desarrollo de la historiografía peruana a lo largo del presente siglo, pocos procedimientos resultan más adecuados que centrar la atención en la vida institucional de la Academia Nacional de la Historia (antes Instituto Histórico del Perú). Durante sus noventa años de existencia, desde la fundación por el Presidente José Pardo en 1905, esta corporación ha reunido a más de un centenar de miembros de número, entre los cuales figuran muchos —ciertamente que no todos-de los más notables exponentes de la disciplina histórica en el país. De tal manera que, a través de la evolución interna de la Academia, es posible rastrear las principales tendencias dentro de las sucesivas generaciones de historiadores peruanos contemporáneos. Con este trabajo queremos brindar un aporte a la interpretación historiográfica, aspecto que ha sido relativamente descuidado en nuestro medio. ${ }^{l}$

\section{Antecedentes}

Los remotos antecedentes de las actuales instituciones académicas del Perú pueden ubicarse en la Academia Antártica, fundada y presidida en Lima por Antonio Falcón, que sirvió de cenáculo a los principales poetas del virreinato entre fines del siglo XVI y principios del XVII. Después seguirían sus pasos otras agrupaciones de carácter literario; pero una preocupación más señalada por la historia del país, su situación presente y los medios para remediarla se encuentra sólo a fines de la decimoctava centuria, cuando empieza a gestarse la conciencia de nacionalidad. Entonces aparece, en 1787, la Academia Filarmónica, en la que Hipólito Unanue, José Rossi y Rubí, José María Egaña, Demetrio Guasque y otros jóvenes de la aristocracia criolla limeña —influidos por la filosofía de la Ilustración - se reunían para tratar de "materias literarias" y "noticias públi-

1 Quisiera expresar mi sincero agradecimiento a los doctores Félix Denegri Luna, José Antonio del Busto Duthurburu, Carlos Deustua Pimentel y Ella Dunbar Temple, de la Academia Nacional de la Historia, por las facilidades que me brindaron en la elaboración de este trabajo. La misma nota de gratitud vale para los colegas César Gutiérrez Muñoz y Luis Alberto Ruiz Ramírez. 
cas". ${ }^{2}$ Al poco tiempo, el conjunto se desintegró. Pero no pasarían muchos meses hasta que, en 1790, se estableciera la célebre Sociedad Académica de Amantes del País, que congregó a más o menos el mismo grupo, junto con Calero y Moreira, Baquíjano y Carrillo y otros "hombres estudiosos y verdaderos amantes de la patria". ${ }^{3}$ Ellos decidieron publicar el Mercurio Peruano, periódico de historia, literatura y noticias públicas, con el objeto de "hacer más conocido el país que habitamos". ${ }^{4}$

Hacia mediados del siglo pasado surgen autores con una inquietud histórica más definida, que constituyen los precursores de la historiografía peruana. Son ellos personajes como Mariano Eduardo de Rivero, científico e investigador de monumentos, Mariano Pagador y, fundamentalmente, José María Córdova y Urrutia, quien en 1844 publicó Las tres épocas del Perú y compendio de su historia, primer intento de delinear una historia general de la patria. ${ }^{5}$

En la segunda mitad del siglo queda ya definitivamente formada la historiografía nacional, con el inicial predominio de la corriente liberal y romántica, que realizó una obra de tipo erudito, de acopio de materiales, pero por lo general carente de profundidad interpretativa. Dentro de este grupo se inscriben principalmente Sebastián Lorente, con sus textos de síntesis, el general Manuel de Mendiburu, que precisó informaciones biográficas acerca del período virreinal, y Mariano Felipe Paz Soldán, quien se fijó en la época posterior a las guerras de independencia. Aparte, destacan Manuel Atanasio Fuentes, Manuel de Odriozola, José Antonio de Lavalle y Carlos Lissón, entre otros. ${ }^{6}$

Es a partir de la década de 1860 cuando, superada la lucha caudillista y la amenaza del dominio español y alcanzada en el país una relativa prosperidad, los hombres de letras empezaron a agruparse en entidades académicas de índole humanística. Dentro de estas instituciones, caracterizadas por el amplio marco de sus intereses culturales, los estudios de historia

2 Hesperióphylo [seud., ¿José Rossi y Rubí?], "Historia de la Sociedad Académica de Amantes del País y principios del Mercurio Peruano", en Mercurio Peruano, I: 7 (Lima, 23 de enero de 1791), ff. 49-52. Véase también Clément, Jean-Pierre: "Indices del Mercurio Peruano, 1790-1795", en Fénix, 26/27, Lima, 1979, pág. 10.

3 Calero y Moreira, Jacinto: Prospecto del papel periódico intitulado "Mercurio Peruano", de historia, literatura y noticias públicas, Lima, 1790, pág. [4].

4 Cit. en Hampe Martínez, Teodoro: "La Revolución Francesa vista por el Mercurio Peruano: cambio político vs. reformismo criollo”, Boletín del Instituto Riva-Agüero, 15, Lima, 1988, pág. 166.

5 Rivera Serna, Raúl: "Historia de la historia”, Historia del Perú, Lima, 1980, págs. 296-297.

6 Pease G. Y., Franklin, comp.: Perú; una aproximación bibliográfica. México, 1979, pág. 29, y Rivera Serna, "Historia de la historia", págs. 299-304. 
ocuparon un lugar preferencial. Bajo tales características se constituyeron la Academia Peruana de Ciencias y Bellas Letras (1867), la Sociedad "Amigos de las Letras" (también de 1867) y el Club Literario, que tuvieron corta vida. Más fecunda fue la labor realizada a su vez por el Ateneo de Lima, cuya comisión de Historia y Geografía se instalaría en octubre de 1885 , manteniéndose luego por varios lustros. ${ }^{7}$

Por otra parte, no faltaron iniciativas tendientes a formar una corporación exclusivamente dedicada al quehacer histórico. Fue Carlos Lissón el primero que, en la memoria que redactó en 1871 como decano de la Facultad de Letras de la Universidad de San Marcos, se refirió a la necesidad de establecer una "Academia de Historia, la que mediante el paciente y prolijo afán de varias generaciones dará los elementos de la historia nacional en todos sus ramos". ${ }^{8} \mathrm{Y}$ el general Mendiburu, aludiendo a las deficiencias que padecía la investigación histórica en la patria, sentenció: "Falta organizar el Archivo Nacional, está por crearse una Academia de Historia, y aún no se ha tratado de tomar de los archivos de España copias de muchos escritos que interesan a la República". ${ }^{9}$ Un paso más adelante fue el que dio el coronel Manuel de Odriozola, erudito y prócer de la Independencia; en 1878, siendo director de la Biblioteca Nacional, se propuso - aunque sin éxito- organizar una Sociedad de Historia del Perú, con el fin de entablar entre los investigadores un diálogo de proyectos y realizaciones, fomentar la compilación y publicación de fuentes documentales y atraer la atención del Estado hacia los estudios del pasado patrio. ${ }^{10}$

Consumada la debacle en la guerra con Chile, los grupos dirigentes de la sociedad resolvieron emprender una profunda investigación de la realidad peruana para tratar de explicarse las causas del desastre producido y conseguir luego la "regeneración" del país. En medio de ese desolado ambiente fue surgiendo un sentimiento nacionalista, deseoso de resaltar los valores más importantes de la cultura, la lengua, las tradiciones, el paisaje. Es dentro de tales circunstancias como debe comprenderse el establecimiento de las primeras entidades académicas que han tenido vigencia per-

7 Cf. el discurso de orden de Alberto Tauro en la sesión solemne del 27 de diciembre de 1955 (conmemorativa del cincuentenario del Instituto Histórico del Perú), publicado en Revista Histórica, XXII, Lima, 1955-56, especialmente en las págs. 27-35.

8 Ibídem, pág. 33.

9 Mendiburu, Manuel de: Prólogo a su Diccionario histórico-biográfico del Perú [1874]. Lima, 1976, págs. 42-43.

10 Cf. Tauro, "Manuel de Odriozola, prócer-erudito-bibliotecario", Documenta, 4, Lima, 1965, págs. 24-25. 
durable en el Perú. Así, el 30 de agosto de 1887 tenía lugar la solemne inauguración de la Academia Peruana de la Lengua, formada gracias al empeño de Ricardo Palma; el 22 de febrero del año siguiente un decreto supremo, firmado por el Presidente Andrés A. Cáceres, fundaba la Sociedad Geográfica de Lima, colocándola bajo la dependencia del Ministerio de Relaciones Exteriores. ${ }^{11}$

Por aquella época aparecía en los medios intelectuales limeños la corriente positivista, grandemente influida por la sociología, y en la cual se distinguieron especialmente Javier Prado, Manuel Vicente Villarán, Alejandro Deustua, Mariano H. Cornejo. En cuanto a la investigación histórica, destacan para entonces los trabajos de Enrique Torres Saldamando, Manuel González de la Rosa, José Toribio Polo y Pablo Patrón, sobre los cuales se ha señalado como característica la creencia de que "demostrar las fuentes empleadas para su tarea intelectual era condición fundamental y garantía de su probidad científica". ${ }^{2}$ La mayor parte de esos personajes, y algunos otros más, serían precisamente los forjadores del Instituto Histórico del Perú.

\section{La Fundación (1905)}

En el marco de su plan de fomento a la instrucción pública, y considerando que "conviene propender a la formación de la historia nacional", el Presidente José Pardo y Barreda, por decreto del 18 de febrero de 1905, fundó el Instituto Histórico del Perú. Como medida complementaria, el 8 de marzo una resolución suprema designó al personal fundador, compuesto de cuarenta individuos, entre miembros natos y de número, los únicos en ser designados por el Gobierno durante la trayectoria del organismo. A continuación, el 31 de marzo, se efectuó la primera sesión, que estuvo presidida por el ministro de Justicia, Culto e Instrucción, Jorge Polar, y en

11 La creación de la nueva Sociedad obedecía al propósito de "fomentar los estudios científicos de aplicación, facilitar la explotación e incremento de los productos naturales del país y crear un centro de datos e informaciones sobre la geografía en general y sobre la especial que interesa a la buena marcha de la administración pública", según el facsímil del decreto supremo del 22 de febrero de 1888, en Boletín de la Sociedad Geográfica de Lima, 97, Lima, ene.-jul. 1978, entre las págs. 46 y 47. Véase también Palacios Rodríguez, Raúl: La Sociedad Geográfica de Lima; fundación y años iniciales, Lima, 1988, págs. 43 ss.

12 Cf. Dager Alva, Joseph: Una aproximación historiográfica a la vida y obra de José Toribio Polo, tesis para optar el título de Licenciado en Historia, Lima, Pontificia Universidad Católica del Perú, Facultad de Letras y Ciencias Humanas, 1996, pág. 117. 
ella se eligió una junta directiva provisional, encabezada por Eugenio Larrabure y Unanue, miembro correspondiente de la Real Academia de la Historia de Madrid.

Dicha elección en favor de Larrabure sería ratificada el 11 de julio siguiente, en la primera junta general, en la que se formó el Directorio definitivo. Finalmente, el 29 de julio de 1905 se llevó a cabo la instalación oficial del Instituto, en una brillante ceremonia realizada en el local de la Cámara de Diputados, a la que concurrieron el Presidente de la República, ministros de Estado, magistrados y representantes del cuerpo diplomático.

Los primeros estatutos, que habrían de normar la vida corporativa por más de medio siglo, fueron promulgados el 10 de julio de 1905. En ellos se especifican las atribuciones del Instituto, "cuerpo que tiene por objeto cultivar y promover el estudio de la historia nacional" (art. $1^{\circ}$ ). Bajo su dependencia se puso al flamante Museo de Historia Nacional, creado el 6 de mayo de 1905, que funcionó inicialmente en el Palacio de la Exposición de Lima, y se acondicionó uno de los salones de dicho establecimiento como sede del Instituto. ${ }^{13}$ Posteriormente trasladaría sus despachos al antiguo local de la Biblioteca Nacional, en la calle Estudios.

Entre los fines que se asignaron a la naciente entidad figuran los siguientes: (1) recoger y conservar ordenadamente manuscritos, libros, cartas geográficas, estampas y otros materiales útiles a la historia del Perú; (2) promover y recompensar la redacción de obras históricas, monográficas o generales, sobre el país; (3) proponer al Gobierno lo que considerase útil para el conocimiento, difusión y progreso de las ciencias históricas; (4) conservar los monumentos nacionales de carácter arqueológico o artístico; (5) supervisar la administración de todos los museos y archivos de la República, en especial del Museo de Historia Nacional y del Archivo Nacional. Observamos, pues, que se encomendó a la institución una tarea muy vasta y realmente difícil de cumplir, que la constituía en el órgano rector de todo lo relativo al quehacer histórico y arqueológico en el país.

De acuerdo con los estatutos (art. $4^{\circ}$ ), el Instituto se componía de cuatro clases de miembros: numerarios, natos, honorarios y corresponsales. En cuanto a los miembros natos — categoría que pronto caería en desuso-, podían resultar tales por los cargos que ejercían en la administración pública o la docencia, o bien por ser "correspondientes en el Perú de la Real Academia de la Historia, de Madrid, y de otras academias o sociedades

13 Tello, Julio C., y Mejía Xesspe, Toribio: "Historia de los museos nacionales del Perú, 18221946”, Arqueológicas, 10, Lima, 1967, págs. 59-62. 
extranjeras dedicadas al estudio de la historia". ${ }^{14}$ En virtud de esa disposición, alcanzaron a integrar las filas de nuestro organismo personajes de la talla de Ricardo Palma, el insigne "tradicionista", y director de la Biblioteca Nacional, Constantino R. Salazar, director del Archivo Nacional, y el ex Presidente Francisco García Calderón, que al final de su vida desempeñaba la rectoría de la Universidad de San Marcos.

De otro lado, entre los miembros honorarios que han pertenecido a la institución cabe mencionar a hombres de letras tan prestigiosos como el español Rafael Altamira, el argentino Roberto Levillier, el ecuatoriano Jacinto Jijón y Caamaño, el boliviano Franz Tamayo y el hondureño Rafael Heliodoro Valle (incorporados todos en 1924), el chileno Agustín Edwards y el argentino José Pacífico Otero (en 1935), el español Salvador de Madariaga y el francés Louis Baudin (en 1947), etc. Y por lo que atañe a los miembros corresponsales, éstos han sido, entre muchos otros: Heinrich Brüning (con sede en Eten), José Toribio Medina y Tomás Thayer Ojeda (en Chile), Arturo Posnansky (en Bolivia), Carlos A. Villanueva (en Francia) y el propio escritor peruano Ventura García Calderón, residente en París, quien fuera incorporado en 1949, cuando se hallaba de visita en Lima.

Tarea verdaderamente ardua debió significar para el Gobierno la designación de una treintena de miembros de número fundadores, pues a comienzos del siglo XX eran realmente pocos los estudiosos aplicados por entero a la investigación histórica. ${ }^{15}$ De ahí se explica que muchos de ellos fueran agentes de la burocracia y del foro, que se habían dedicado a la historia de manera sólo incidental. Con todo, en el elenco de fundadores se encuentran varias de las figuras intelectuales y políticas más importantes de principios de siglo: José Sebastián Barranca, Mariano H. Cornejo, Emilio Gutiérrez de Quintanilla, Miguel Antonio de la Lama, Víctor M. Maurtúa, Javier Prado, Nemesio Vargas, Carlos Wiesse...

Según lo estipulado en los estatutos (art. $5^{\circ}$ ), mientras que las plazas para honorarios y corresponsales eran ilimitadas, los miembros de número no podían exceder de treinta. Cuando se producía alguna vacante, el nuevo postulante - propuesto por tres numerarios antiguos - debía ser aceptado mediante votación en el Directorio, primero, y en la junta general después.

14 Cf. Hampe Martínez, T.: "Los miembros de número de la Academia Nacional de la Historia (Instituto Histórico del Perú), 1905-1984”, Revista Histórica, XXXIV, Lima, 1983-1984, pág. 282.

15 Sobre las dificultades que todavía hoy afrontan investigadores y docentes para mantenerse con su trabajo profesional en historia, véase Bronner, Fred: "Peruvian historians today: historical setting”, The Americas, 43: 3, Washington DC, ene. 1987, págs. 245-277. 
Una vez electo, estaba obligado a tomar posesión de su asiento en el término de tres meses, exponiendo en acto público un trabajo inédito. Sin embargo, varias de estas disposiciones perdieron vigencia en breve, y la práctica simplificó el trámite de ingreso a tal punto que por muchos años se eliminó el requisito de la ceremonia especial de incorporación.

Con el transcurso del tiempo, se han sucedido en las plazas del Instituto Histórico (convertido luego en Academia Nacional de la Historia) más de una centena de investigadores, hermanados por su común dedicación al pasado peruano. A través de ellos es posible reconocer las características más relevantes de cada una de las generaciones de historiadores de nuestro siglo, teniendo en cuenta que - tal como anota César Pacheco Vélez, citando a Ortega y Gasset - cada generación implica un modo peculiar de ver el mundo, de sentir la existencia e interpretar la historia. ${ }^{16}$ Examinando la relación de los presidentes de la corporación, de los miembros de número y de los trabajos publicados en la Revista Histórica, su órgano oficial, es factible extraer conclusiones bastante certeras respecto a la intervención que han tenido diferentes ideologías, tendencias historiográficas e intereses político-económicos en la marcha de este organismo.

\section{Primera época (1905-1945)}

Son los integrantes de la generación positivista quienes fundan el Instituto Histórico y retienen en él los cargos directivos por espacio de cuatro décadas, en lo que representa la primera época de la corporación. Desde el punto de vista historiográfico, el positivismo aporta — como es sabidouna renovación de métodos y criterios, que se manifiesta en mayor rigor heurístico y en la introducción de consideraciones sociológicas en el estudio del pasado. ${ }^{17} \mathrm{El}$ dominio de los positivistas dentro de la marcha del Instituto se deja percibir nítidamente a través de varios factores.

En primer lugar, componentes de dicha generación, vinculados políticamente al civilismo, ocuparon la presidencia en forma ininterrumpida has-

16 Pacheco Vélez, César: "75 años de historia del Perú”, La Prensa, LXXV: 32.519, Lima, 22 de setiembre de 1978, pág. 9 .

17 Cf. Pacheco Vélez: "La historiografía peruana contemporánea", en Pareja Paz-Soldán, José, comp.: Visión del Perú en el siglo XX, Lima, 1963, II, págs. 530-532. Véase también el estudio introductorio de C. P. Gooch a Leopold von Ranke: Pueblos y estados en la historia moderna, México, 1948, especialmente las págs. 32-33. 
ta 1946. Durante esos años dirigieron la institución Eugenio Larrabure y Unanue (1905-1916), el general Juan Norberto Eléspuru (1916-1921), Felipe de Osma y Pardo (1921-1924), Emilio Gutiérrez de Quintanilla (vicepresidente, encargado del mando en 1924-1926) y Mariano Ignacio Prado y Ugarteche (1926-1946). Además, muchos de los miembros fundadores eran destacados representantes de la corriente positivista en el Perú; tales son los casos de M. H. Cornejo, J. Prado, C. Wiesse y varios otros, a los cuales se agregarían años después eruditos investigadores como Manuel González de la Rosa, Rómulo Cúneo Vidal, Horacio H. Urteaga y Luis Antonio Eguiguren. De otro lado, la dirección de la Revista Histórica estuvo casi todo ese tiempo en manos de Carlos A. Romero, numerario fundador, exponente también del grupo positivista, quien se encargó de dar a luz los tomos I a XVI (entre 1906 y 1943).

Para comprender los contornos de notoriedad que alcanzara el organismo en su primera época, es necesario considerar que ésta coincide con lo que puede entenderse como la "primacía del historicismo" en el Perú, que abarca desde los últimos tramos del siglo pasado hasta el fin de la Segunda Guerra Mundial. Como bien advierte Pablo Macera, durante el referido lapso la historia significó en nuestro país la ciencia social por excelencia; brindaba un tipo de conocimiento que servía de modelo a las demás disciplinas y del cual se buscaba obtener una herramienta de acción social, una técnica para operar racional y eficazmente sobre el quehacer humano. Se dio entonces una conjunción entre historia y política, se manejó la historia como un instrumento destinado a averiguar las raíces de la formación económico-social del presente y sus proyecciones hacia el porvenir. ${ }^{18}$

En medio de ese contexto, el Estado favoreció enormemente el desarrollo de los estudios históricos, sobre todo durante el "oncenio" de Augusto B. Leguía. Tal apoyo se verificó de diversas maneras: por medio de las celebraciones alusivas al centenario de la Independencia y de la batalla de Ayacucho, que ocasionaron la realización de certámenes internacionales; la reestructuración del Archivo Nacional, puesto bajo la dirección de Urteaga a partir de 1919; la fundación del Museo Bolivariano y de repositorios arqueológicos, tanto en la capital como en provincias; la reforma

18 Macera, Pablo: "La historia en el Perú: ciencia e ideología" [1968], en sus Trabajos de historia, Lima, 1977, I, págs. 4-5. En el mismo sentido, Ernesto Yepes opina que durante la fase del segundo civilismo (1895 a 1920) la historia sirvió en el Perú como un razonamiento acerca de la sociedad, explícitamente comprometido con un orden y con el proyecto político de una larvaria burguesía no industrial, especulativa, comercial y agro-exportadora; véase "La investigación de la historia social en el Perú”, Historia y sociedad en el Perú del siglo XX, La Molina [Lima], 1978, I, pág. 3. 
universitaria de 1928, que aumentó el número de cursos de Historia del Perú, etc. ${ }^{19}$

Pero las nuevas corrientes de pensamiento originadas en los países desarrollados de Europa no tardarían en causar su impacto en el Perú. En efecto, y de acuerdo con el planteamiento de Guillermo Lohmann Villena, será decisiva la realización en Lima del XXVII Congreso Internacional de Americanistas (1939) y de la III Asamblea General del Instituto Panamericano de Geografía e Historia (1941), ya que permitieron a los estudiosos del medio capitalino tomar contacto directo con los modelos del exterior. ${ }^{20}$ Tan es así que a partir de los años 1940, como reflejo de las concepciones económico-sociales de la escuela francesa de los Annales, se registrará un movimiento orientado a la revisión de los postulados hasta entonces vigentes en la disciplina histórica, que pasarían a llamarse "tradicionales". En consecuencia, queda relativamente liquidada la primacía del historicismo, y la iniciativa dentro de las ciencias sociales se traslada de la historia a la antropología, primero, y luego a la sociología y la economía. ${ }^{21}$

A la vez que las nuevas corrientes doctrinarias ejercían su efecto, principalmente entre los historiadores jóvenes, se hacía más patente la escasa actividad desarrollada por el Instituto Histórico, una entidad de carácter académico-ceremonial y limitada casi exclusivamente a la edición de la Revista Histórica. Esto resultaba tan evidente que, en un discurso de 1950, Lohmann Villena — quien más tarde sería presidente de la entidad- llegó a criticarla airadamente en los términos siguientes: "Pero la vida de una institución no es simplemente la publicación esporádica de un boletín. El Instituto Histórico ha estado sumido en un sueño que le ha mantenido ausente de nuestros grandes acontecimientos conmemorativos, de las controversias nacionales y de la vida corporativa....".22

Dentro de tales circunstancias, el año 1945 se presenta como una barrera crucial, que determina el fin de la primera época del Instituto. En el terre-

19 Cf. Lohmann Villena, Guillermo: "Los estudios históricos en el Perú en los últimos cincuenta años", Mercurio Peruano, XXV: 282, Lima, set. 1950, págs. 330-331 y 333.

20 Ibídem, pág. 339.

21 Este proceso ha sido inteligentemente abordado en el ensayo de Burga, Manuel: "Los Annales y la historiografía peruana (1950-1990): mitos y realidades", Ciencias Sociales, 1, Lima, 1995, págs. 11-33. Sugiere dicho autor que una serie de factores de orden intelectual, político y social facilitaron la permeabilidad de los historiadores peruanos a la nueva escuela histórica francesa: “...el espíritu iconoclasta, la actitud herética, la convicción científica globalizante y la posibilidad de un acercamiento sistemático a las tradiciones culturales nacionales, que permitía y, de alguna manera, promovía la escuela de Annales" (pág. 31).

22 Lohmann Villena: "Los estudios históricos en el Perú", pág. 327. 
no político constituye un sugestivo momento de apertura democrática, permitiendo el acceso al poder del Frente Democrático Nacional, con la candidatura (de ancha base representativa) de José Luis Bustamante y Rivero, y en el ámbito de la cultura marca el florecimiento de la vida institucional. Para empezar, el 22 de julio de 1945 una docena de jóvenes catedráticos y egresados universitarios - reunidos por la diligente iniciativa de Ella Dunbar Temple - suscribían el acta fundacional de la Sociedad Peruana de Historia. Esta, que ha subsistido hasta hoy paralelamente a la Academia, introdujo en aquel momento una renovación en las concepciones científicas; propugnaba, por vez primera, "estimular la profesión de historiador" y marcaba el comienzo del trabajo multidisciplinario en equipo, promoviendo la especialización de los investigadores en áreas específicas de las ciencias humanas. ${ }^{23}$

También en 1945 inauguraron sus actividades otras agrupaciones ligadas al cultivo de la historia, como el Centro de Estudios Histórico-Militares y el Instituto Peruano de Investigaciones Genealógicas. Al año siguiente se establecía en la Universidad de San Marcos el Instituto de Etnología, gracias al fecundo impulso de Luis E. Valcárcel. Y en mayo de 1947 quedaba fundado - bajo la conducción de Víctor Andrés Belaunde- el Instituto Riva-Agüero, centro de altos estudios adscrito a la Pontificia Universidad Católica del Perú, dedicado esencialmente a la investigación histórica, arqueológica, lingüística y filosófica.

\section{Segunda época (1945-1962)}

El advenimiento de la segunda época está signado además por un nefasto acontecimiento, el incendio de la Biblioteca Nacional en mayo de 1943, que dejó a la entidad con un par de graves problemas: la falta de local y la destrucción de sus documentos oficiales. Por lo que atañe al primer aspecto, salvo un breve intervalo en el que funcionó en el antiguo colegio de San Pedro, el Instituto careció de sede, debiendo los miembros reunirse en lugares como el Museo Nacional de Historia y las residencias de diferentes directivos, hasta que el 17 de julio de 1963 se tomó posesión de una sala que le fue asignada en la casona de Pilatos, convertida en establecimiento de la Casa de la Cultura. Sin embargo, el problema no ha quedado satisfactoriamente resuelto, y aún persiste la búsqueda de una ubicación más adecuada.

23 Cf. Pacheco Vélez: "La historiografía peruana contemporánea”, págs. 563-564. 
De otro lado, el 28 de junio de 1945, al reiniciarse las labores tras el incendio, el prosecretario Salvador Romero Sotomayor informaba sobre la desaparición de los libros de actas — que habían estado a su cargo- como consecuencia del siniestro. ${ }^{24}$ Pero, felizmente, "se ha podido recuperar una carpeta con actas y minutas de las sesiones del Instituto Histórico en 1921 y 1922, que se creían perdidas en el incendio de la Biblioteca Nacional y que contribuyen a facilitar la continuidad de las labores", según manifestaba Aurelio Miró Quesada, presidente del organismo, en el discurso-memoria que leyó el 24 de enero de $1964 .{ }^{25}$ Este mismo día el académico Luis Alayza y Paz Soldán, refiriéndose a dichas actas, correspondientes al tiempo de la presidencia del general Eléspuru, argumentó "en el sentido de que se publicaran en la Revista de la Academia, ya que constituían un documento de valor para la institución en su segundo decenio". ${ }^{26}$ Hasta la fecha, sin embargo, no se ha llevado a efecto esa valiosa propuesta.

Desaparecidos físicamente los representantes del positivismo e iniciada una nueva etapa en la existencia del Instituto Histórico, son los exponentes de la generación del novecientos quienes asumirán la dirección. En su obra, en general, está latente una inquietud intelectual de firme sustento patriótico: intentan conseguir la "regeneración" de la patria mediante el estudio paciente y sistemático de su realidad, presente y pasada. En cuanto a lo historiográfico, dicha generación superó criterios mecanicistas del positivismo y se adhirió al neohumanismo espiritualista, dándole mayor animación a la evocación histórica; aportó una visión integral de la historia del Perú, que entendió como una nación mestiza y cristiana, síntesis de lo indígena con lo hispánico. Los miembros de este grupo produjeron una renovación sustantiva en la historiografía patria, y han tenido honda y fecunda trascendencia hasta la actualidad..$^{27}$

Los "novecentistas" comienzan a participar en la marcha de la institución desde los primeros años, cuando apenas egresaban de las aulas universitarias. Figuras como Víctor Andrés Belaunde y Felipe Barreda y Laos (1908),

24 Libro de actas del Instituto Histórico del Perú; segunda época (comienza el 28 de junio de 1945), pág. 4. Se trata de un volumen empastado, de 400 páginas, con notas manuscritas de todas las sesiones que ha celebrado la institución desde esa fecha.

25 Publicado en Revista Histórica, XXVI, Lima, 1962-1963, págs. 432-440; cita de las págs. 433-434.

26 Libro de actas del Instituto Histórico del Perú, págs. 161-162.

27 Pacheco Vélez, comp.: "Los historiadores del Perú en la generación del 900”, Fanal, XIX: 69, Lima, 1964, págs. 2-11, y Hampe Martínez, T.: "El P. Vargas Ugarte y su aportación a la historiografía del Perú colonial”, Revista de Historia de América, 104, México, jul.-dic. 1987, págs. 147-148. 
José de la Riva-Agüero y Juan Bautista de Lavalle (1911), José Gálvez Barrenechea (1919) y Oscar Miró Quesada (1920) se incorporan a la entidad durante sus tres primeros lustros; posteriormente se agregarán Rubén Vargas Ugarte, Luis Alayza, Julio C. Tello y Francisco García Calderón. Sin embargo, la oposición política de casi todos ellos al gobierno de Leguía los obligaría a vivir desterrados del país hasta 1930, por lo menos. Este hecho determinó su alejamiento de los puestos rectores de la vida académica nacional, que permanecieron largo tiempo en poder de las generaciones mayores.

En 1946, a la muerte de Mariano Ignacio Prado, que había regido el organismo a lo largo de dos décadas, se hizo cargo de la presidencia Víctor Andrés Belaunde. Pero se mantuvo brevemente en el puesto, ya que - ocupado en múltiples labores diplomáticas e intelectuales-optó por renunciar en enero de 1948, para ser sucedido por el primer vicepresidente, Alayza; éste a su vez traspasó el mando al cabo de 22 meses, en noviembre del año siguiente, a Oscar Miró Quesada. Bajo la gestión de Racso, el ilustre periodista, escritor y hombre de ciencia, la institución recobró impulso, consiguiéndose en 1952 un incremento en el apoyo económico del Estado, que pasó de 500 soles (monto vigente desde la fundación) a 4.000 soles anuales. ${ }^{28}$ Tocóle a Miró Quesada organizar la conmemoración del cincuentenario del Instituto, que se efectuó el 27 de diciembre de 1955 en un acto solemne en el auditorio de la Biblioteca Nacional, en presencia del ministro de Educación Pública y otras autoridades del ramo; el discurso de orden estuvo a cargo de Alberto Tauro, quien disertó sobre la enseñanza y la investigación histórica en el primer siglo de la República.

La generación del Centenario (o de la Reforma Universitaria), surgida alrededor del famoso Conversatorio sanmarquino de 1919, evidencia - como puntualiza con acierto Macera - un cambio en la composición social de la historiografía peruana: los grupos de poder tradicionales son sustituidos por capas medias. Esto queda claro, por ejemplo, en los casos de Raúl Porras Barrenechea (proveniente de una familia aristocrática pero empobrecida), Jorge Guillermo Leguía, Luis Alberto Sánchez y Jorge Basadre, que estuvieron entre los principales animadores de aquel Conversatorio. ${ }^{29} \mathrm{En}$ su obra de investigación, que nace bajo cierto matiz socialista, se aprecia una inquietud más explícita por estudiar los factores econó-

28 Acta de la junta directiva del 9 de julio de 1952, en Libro de actas del Instituto Histórico del Perú, pág. 68.

29 Macera, Pablo: "La historia en el Perú", págs. 7-8, y "Explicaciones", en sus Trabajos de historia, Lima, 1977, I, pág. XXV. 
micos y hay un avance en el rigor heurístico e interpretativo; al mismo tiempo, comienza la formación de escuelas históricas, con una dedicación más exclusiva a esta disciplina. ${ }^{30}$

Debido a lo novedoso de sus planteamientos, empero, el grupo de la Reforma no encontró cabida en el Instituto Histórico más que a partir de la segunda época. Aunque un par de representantes "de avanzada" como L. A. Sánchez y L. E. Valcárcel se habían incorporado ya en 1928 y 1932, respectivamente, sólo desde 1945 empieza a ingresar el grueso de esta generación. Entonces quedan integrados, uno tras otro, Porras, Basadre, Manuel Moreyra, Aurelio Miró Quesada — estos dos últimos distanciados de los postulados originales del grupo reformista, pero se vinculan a él cronológicamente-, Emilio Romero, Alberto Ulloa, José Luis Bustamante y Rivero. El grupo innovador del Conversatorio alcanzó la presidencia de nuestro organismo en julio de 1956, por intermedio de Jorge Basadre, quien habría de mantener el cargo por espacio de seis años, en lo que representa sin embargo una fase de relativamente escasa actividad.

\section{Tercera época: Academia (desde 1962)}

No obstante que los primeros estatutos se mantenían vigentes desde la fundación en 1905, el Instituto Histórico había experimentado con el paso del tiempo una serie de modificaciones en su estructura. Primeramente, muy pronto cayó en desuso la disposición referente a los miembros natos, que dejaron de ser incluidos. En cuanto a atribuciones, se restringió en 1911 la directa injerencia que el Instituto tenía sobre la administración del Museo de Historia Nacional, que quedó limitada a la tarea de inspección; veinte años después, cuando se reorganizó la política cultural de la República, dicho Museo obtuvo plena autonomía. ${ }^{31}$ Por otra parte, el 25 de julio de 1920 el Instituto era reconocido como correspondiente por la Real Academia de la

30 Es un hecho evidente que el oncenio de Leguía, que marca el fin de la llamada República Aristocrática, significa una ruptura en el desarrollo político peruano. Se produce entonces el ingreso al escenario del poder de nuevas fuerzas sociales, como las clases medias y obreras, hecho que conlleva la formulación de alternativas ideológicas, encaminadas a transformar en plazo más o menos breve la estructura de la sociedad. Dentro de este contexto surgen, pues, el pensamiento socialista de Mariátegui y el aprista de Haya de la Torre, con su respectiva incidencia en el quehacer historiográfico. Véase Caravedo Molinari, Baltazar: "La investigación histórica en el Perú: contexto social y lineamientos teóricos", Cuadernos del Consejo Nacional de la Universidad Peruana, 20/21, Lima, ene.jun. 1976, págs. 14-15.

31 Cf. Tello y Mejía Xesspe: "Historia de los museos nacionales...”, págs. 181-189. 
Historia de Madrid, encargándose entonces la presidencia a Felipe de Osma y Pardo, dado que era el más antiguo de los miembros corresponsales de la Academia madrileña en el Perú. Se decidió inclusive reducir progresivamente la cantidad de plazas de número a dieciocho, a fin de igualarse con la corporación española, pero tal medida no llegaría a tener efecto.

Una resolución suprema emanada del Ministerio de Educación Pública el 24 de diciembre de 1962 aprobó los nuevos estatutos de la institución, derogando los que estaban en vigor desde principios de siglo. Dicho texto, que había comenzado a prepararse durante la administración de Basadre, señala en su artículo $1^{\circ}$ que "el Instituto Histórico del Perú [...] es la Academia Nacional de la Historia, que tiene por objeto cultivar y promover el estudio de la historia patria y de los problemas conectados con las ciencias históricas". ${ }^{32}$ Tal cambio de denominación sería confirmado mediante un decreto supremo del 18 de setiembre de 1964, suscrito por el Presidente Fernando Belaunde Terry, que reconoce el moderno apelativo de Academia Nacional de la Historia (Instituto Histórico del Perú).

Entre los cometidos encargados al organismo a través de los nuevos estatutos (art. $2^{\circ}$ ) figuran los siguientes: (1) reunir y organizar materiales, éditos e inéditos, de interés para la historia del Perú y hacer que éstos sean conservados en sus originales o copias; (2) publicar colecciones de fuentes documentales, obras raras o inéditas que contribuyan a divulgar y esclarecer la historia nacional; (3) cuidar la fidelidad de los textos de Historia del Perú, pudiendo presentar observaciones para que el Estado, mediante sus órganos competentes, adopte las medidas tendientes a dicho fin; (4) proporcionar los informes que le soliciten los poderes del Estado; (5) supervigilar los archivos y museos del país; (6) intervenir con su dictamen en la nomenclatura de las calles y plazas de la República. En cuanto a los académicos, quedó oficializada la supresión de los miembros natos y se ratificó el límite de treinta numerarios — profesionales con residencia habitual en Lima-, volviéndose a insistir en el requisito del discurso de incorporación, si bien el plazo respectivo se amplió a los seis meses siguientes a la elección.

Dentro de la tercera época del organismo, que se prolonga hasta nuestros días, destaca nítidamente la gestión realizada por Aurelio Miró Quesada. Este notable crítico e investigador, a quien por su ubicación cro-

32 Los estatutos están publicados en Revista Histórica, XXVI, Lima, 1962-1963, págs. 441447. A nivel interno, el proyecto de nuevos estatutos había sido aprobado en la junta general del 12 de diciembre de 1962; cf. Libro de actas del Instituto Histórico del Perú, págs. 100-113. 
nológica se le puede considerar epígono de la generación del Centenario, llevó adelante las gestiones oficiales encaminadas a brindar nueva fisonomía a la entidad, y fue elegido primer presidente de la Academia Nacional de la Historia el 25 de enero de 1963. Desde entonces, y durante exactamente cuatro años, desarrolló una profusa actividad, contribuyendo eficazmente a elevar el prestigio de la corporación; las sesiones se efectuaron a ritmo intenso, las plazas de número estuvieron enteramente cubiertas, se aplicó la obligación de realizar ceremonias especiales para incorporar a los nuevos académicos, la Revista Histórica apareció regularmente cada año y - como ya hemos adelantado - se consiguió un local propio. Además, del 15 al 24 de setiembre de 1965 tuvo lugar el Congreso sobre el Mestizaje, donde intervinieron numerosos estudiosos nacionales y extranjeros, en conmemoración del sexagésimo aniversario de la fundación del Instituto Histórico.

En 1967, con el acceso de Guillermo Lohmann Villena a la presidencia, se inaugura el predominio de la llamada generación de la Sociedad Peruana de Historia en la vida institucional. Los integrantes de esta generación - conocida también con el renombre de "clausurada"- realizaron su formación universitaria en los años siguientes a la caída de Leguía, época caracterizada por la supremacía política de militares y conservadores, lo cual habría de generar en ellos la presencia de criterios y valoraciones tradicionales al estudiar el pasado, así como un retraimiento instrumental (heurístico), manifestado sobre todo en trabajos de índole bibliográfica y documental. ${ }^{33}$ Miembros de la Sociedad Peruana de Historia han participado desde jóvenes en el seno de la Academia, publicando trabajos en la Revista Histórica y ocupando plazas de número; tal es el caso de G. Lohmann, A. Tauro y E. D. Temple, que fueron incorporados entre 1946 y $1948 .^{34}$ Algunos años más tarde ingresarían también a las filas académicas otros fundadores y miembros de los años iniciales de la Sociedad, como Jorge C. Muelle (1955), José A. de la Puente Candamo (1960) y Bolívar Ulloa Pasquette (1963).

33 Macera: "La historia en el Perú", págs. 11-12, y Yepes: "La investigación de la historia social en el Perú", pág. 17.

34 El caso de Lohmann Villena es algo especial, pues fue designado primero miembro correspondiente (con residencia en España) el 5 de julio de 1946, y sólo accedió a la categoría de miembro de número el 20 de mayo de 1955; según el Libro de actas del Instituto Histórico del Perú, págs. 15 y 73-74. Por otra parte, hay que considerar en este mismo marco generacional a Félix Denegri Luna, quien, no obstante nunca haber pertenecido a la Sociedad Peruana de Historia, se adscribe cronológicamente a los fundadores de ella. 
Los doce años del mandato de Lohmann Villena, de 1967 a 1979 —en coincidencia casi exacta con el gobierno revolucionario de las Fuerzas Armadas-, significan una de las etapas de mayor inacción en la historia del organismo. A partir de entonces faltó casi absolutamente el apoyo estatal. Pese a todo, en dicho lapso se incorporaron varios investigadores pertenecientes a las más jóvenes generaciones de la historiografía nacional, configurando de este modo la actual estructura de la Academia. Aquí distinguimos la sólida presencia de la denominada generación del medio siglo, cuyos integrantes - repartidos entre los claustros de la Católica y San Marcoshan tratado de superar la clásica historia narrativa, de sucesos políticos y militares, incluyendo esquemas de análisis económico y social. ${ }^{35}$

Al lado de historiadores propiamente dichos, pertenecen a la generación del medio siglo varios antropólogos y arqueólogos. Sus primeros representantes - César Pacheco Vélez, Carlos Deustua Pimentel, José Antonio del Busto- empezaron a colaborar en la Revista Histórica a mediados de la década de 1950, e ingresaron como miembros de número en nuestra corporación a partir de 1967. Por otra parte, también han llegado a incluirse contribuyentes de las más jóvenes y radicales tendencias historiográficas, con una orientación de base inicialmente marxista, aportando modernos métodos de investigación, nuevos temas y enfoques interpretativos. Aquí se comprenden las tendencias de la etnohistoria andina, la historia social y económica y la perspectiva de las mentalidades, que pueden verse representadas a través de Franklin Pease G. Y. (desde 1980), Scarlett O'Phelan Godoy y Fernando de Trazegnies Granda (desde 1995), respectivamente. ${ }^{36}$

Gracias al impulso de Félix Denegri Luna, elegido para la presidencia el 15 de junio de 1979, la Academia Nacional de la Historia reanudó sus tareas después de un prolongado letargo. Por lo menos en los inicios de su gestión, Denegri se mostró empeñado en reanimar la vida institucional de diversas maneras: cubriendo parcialmente las numerosas plazas vacantes, reiniciando la publicación de la Revista y organizando el coloquio internacional "Túpac Amaru y su tiempo", que se llevó a cabo en las ciudades de Lima y Cuzco en 1980, por el bicentenario de la gran rebelión andina. Asimismo, cabe anotar que el 31 de octubre de 1980 se celebró el $75^{\circ}$ ani-

35 Pacheco Vélez: "La historiografía peruana contemporánea", págs. 568-570.

36 Cf. Macera: "Explicaciones", págs. LVIII-LX y LXIV. Véase también Bonilla, Heraclio: "The new profile of Peruvian history", Latin American Research Review, 16: 3, Abuquerque, NM, 1981, págs. 210-224, y Pease G. Y., Franklin: "Historia en el Perú del siglo XX", Cuadernos de la Facultad de Letras y Ciencias Humanas [PUCP], 5, Lima, 1992, págs. 7-22. 
versario de la fundación del Instituto Histórico, en una sesión pública realizada en el Museo Nacional de Historia, con asistencia del Presidente de la República y de otros prominentes dignatarios (y donde el académico José A. de la Puente Candamo tuvo a su cargo la disertación central).

Una nueva etapa en la trayectoria institucional se ha abierto el 13 de julio de 1995 con la elección del mencionado catedrático de la Universidad Católica, J. A. de la Puente Candamo, para el cargo de presidente. Se trata de un reconocido investigador de nuestro período de la Independencia, por larguísimos años director del Instituto Riva-Agüero, donde ha sido forjador de varias generaciones de historiadores noveles y ha defendido tenazmente la idea del ser mestizo de la nacionalidad peruana. ${ }^{37}$ Sobre sus hombros recae la responsabilidad de asegurar el mantenimiento de la augusta corporación y guiarla hacia el umbral del tercer milenio.

\section{Balance y perspectivas}

En total, son 110 los miembros de número que han pertenecido a la Academia Nacional de la Historia en sus noventa años de funcionamiento (cuya nómina puede verse en el Apéndice, infra). De ellos, por lo que atañe a su profesión, la mayoría han sido abogados, aunque también ha habido bastantes diplomáticos, economistas, funcionarios, educadores e ingenieros, así como sacerdotes y militares; por cierto que la dedicación exclusiva a la historia, concebida como tarea profesional, es un fenómeno únicamente de los años más recientes. Al seno de la institución se han integrado tres mujeres, brillantes investigadoras del pasado andino prehispánico y colonial, que son Ella Dunbar Temple (electa en 1946), María Rostworowski de Diez Canseco (1979) y Scarlett O'Phelan Godoy (1995). Por otra parte, ha habido un solo extranjero: Max Uhle, el arqueólogo sajón, que prestó servicios al Estado peruano entre 1906 y 1911.

Respecto a la edad de los miembros, indicamos ya previamente que los más jóvenes al incorporarse fueron los componentes de la generación del novecientos, quienes desde temprano recibieron el apoyo de los intelectuales mayores. De esta manera, Felipe Barreda y Laos ingresó cuando apenas contaba 21 años, en tanto que Juan Bautista de Lavalle lo hacía a los 24,

37 Cf. Hampe Martínez, T.: "José A. de la Puente Candamo en la historiografía peruana", Boletín del Instituto Riva-Agüero, 12, Lima, 1982-1983, especialmente las págs. 153-157 y 160-163. 
Víctor Andrés Belaunde a los 25, José de la Riva-Agüero a los 26; asimismo, se incorporaron relativamente jóvenes Luis Varela y Orbegoso (27 años), el padre Domingo Angulo (28 años) y Luis Alberto Sánchez (28 años). En el otro extremo, el de los más ancianos, corresponde la primera mención a Felipe Cossío del Pomar, el pintor y crítico de arte, quien fuera elegido numerario casi en vísperas de su muerte, a los 93 años. Le siguen en orden de edad Modesto Basadre y Chocano, miembro fundador que declinó el nombramiento (89 años), el embajador Juan Miguel Bákula Patiño (81 años), el general Felipe de la Barra (76 años), José Sebastián Barranca (75), José Luis Bustamante y Rivero (74), Alberto Ulloa Sotomayor (73), etc.

En cuanto a la permanencia dentro de la institución, los contrastes son tanto o más pronunciados. Mientras que hubo tres miembros fundadores - Teodorico Olaechea, José Román de Idiáquez y Manuel Jesús Obín-que no completaron siquiera un año en el puesto (fallecieron a los dos, cinco y siete meses de su designación, respectivamente), se ha dado el caso de Luis Alberto Sánchez, el prolífico escritor y político aprista, que conservó su asiento durante 66 años: prácticamente toda una vida. Otros miembros que se han mantenido largo tiempo dentro de la Academia son Felipe Barreda y Laos (65 años), Oscar Miró Quesada (61 años), Juan Bautista de Lavalle (59 años), Víctor Andrés Belaunde (58 años) y Luis E. Valcárcel (55 años).

Revisando la lista de miembros de número, se observa la reiteración de determinados apellidos, de conocida prosapia limeña, como demostración de cercanos lazos de parentesco. Efectivamente, entre Eugenio y Carlos Larrabure, Luis y Bolívar Ulloa, Nemesio y Rubén Vargas, se da una vinculación paterno-filial. Además, no faltan las relaciones entre hermanos (como Javier y Mariano I. Prado), entre sobrino y tío (Aurelio y Oscar Miró Quesada), entre nieto y abuelo (los homónimos José Agustín de la Puente), entre primos hermanos, etc. Tal complejo de redes familiares ilustra bien claramente la existencia de un núcleo reducido, una élite intelectual, que es la que en gran parte ha llevado adelante los estudios históricos y la vida académica en el Perú republicano.

Hemos efectuado, pues, un recuento de los personajes y los hechos más relevantes en la vida de la Academia Nacional de la Historia, a lo largo de sus noventa años de existencia. Se percibe que los valores fundamentales de su obra consisten en haber editado la Revista Histórica, importantísima fuente de consulta para toda clase de investigaciones acerca de la historia peruana, y en haber congregado a muchos de los más notables 
exponentes del quehacer histórico en el país. Sin embargo, como siempre ocurre con este género de corporaciones, se puede discutir bastante en torno a la ausencia - y presencia - en la Academia de variados personajes, tanto de ayer como de hoy.

Por lo que se refiere a los ya fallecidos, podemos señalar con lástima la falta de estudiosos como Germán Leguía y Martínez, Pedro Dávalos y Lissón, Jorge Guillermo Leguía, entre otros. En cuanto a los de la actualidad, la lista podría alargarse notablemente, pero bastará con mencionar el ejemplo de Pablo Macera, el maestro sanmarquino, autor de fundamentales trabajos sobre la vida económica, social y artística de la Colonia, cuyo nombre fuera postulado en 1970 por Guillermo Lohmann, entonces presidente de la institución, para ocupar una plaza de académico; la propuesta, sin embargo, no obtuvo acogida. ${ }^{38}$ Con miras al futuro, será pertinente insistir en que sólo abierta a diferentes corrientes de pensamiento, y desarrollando plena actividad en la docencia y la investigación, se constituirá la Academia Nacional de la Historia en lo que efectivamente le corresponde ser: el principal cuerpo dentro de los estudios históricos en el Perú.

No estará demás añadir unas someras reflexiones sobre el carácter de la historiografía nacional. Es evidente que en el correr del siglo XX ha habido progresos en cuanto a la metodología y complejidad del análisis, buscando dar mayor profundidad a la comprensión de los fenómenos históricos; pero también es cierto que aún falta remediar algunas serias deficiencias. En primer lugar, la excesiva inclinación monográfica ha impedido realizar trabajos de síntesis, obras situadas en un plano intermedio entre los textos elementales y la investigación erudita, que ofrezcan una visión global, panorámica, de la historia patria. Igualmente, hacen falta esquemas interpretativos surgidos de nuestra propia realidad, pues lo constante ha sido la importación de (y la dependencia respecto a) herramientas teóricas originadas en otros países, cuya aplicación en el medio local resulta muchas veces discutible. Y de la moderna proliferación de entidades culturales, tanto públicas como privadas, habrá que esperar —ojalá- la formación de auténticas escuelas, equipos de trabajo, grupos de investigadores vinculados por una inquietud científica común.

38 Acta de la junta directiva del 22 de octubre de 1970, en Libro de actas del Instituto Histórico del Perú, pág. 321. Respecto al indudable valor de la contribución de dicho intelectual se ha escrito: "Dotado de una sólida formación profesional, de una mente ágil, crítica, y de una pluma incisiva, Pablo Macera [...] es probablemente el historiador que más influencia ha ejercido sobre los jóvenes estudiosos que a partir de la década del sesenta irrumpen desde diversas disciplinas en el razonamiento histórico peruano" (Yepes: "La investigación de la historia social en el Perú", pág. 22). 


\section{Apéndice}

MIEMBROS DE NÚMERO DE LA ACADEMIA NACIONAL DE LA HISTORIA (INSTITUTO HISTÓRICO DEL PERÚ), 1905-1995

1. Luis Alayza y PAZ Soldán (1883-1976) 1940

2. Félix Alvarez Brun (n. 1922) 1979

3. Domingo Angulo Mendoza, O.P. (1879-1941) 1907

4. Ricardo ARANDA VARGaS-MachuCa (1849-1922) 1916?

5. Mons. Manuel Segundo BAllón ManRiQue (1854-1923) -

6. Juan Miguel BáKUla PATiÑo (n. 1914) 1995

7. Gral. Felipe de la Barra Ugarte (1888-1978) 1963

8. José Sebastián BARRANCA LOVERA (1830-1909) 1905

9. Felipe BARREDA y LAOS (1888-1973) 1909

10. Víctor Manuel Barriga Velarde, O. de M. (1890-1955) 1945

11. Modesto BASADRE y ChOCANO (1816-1905) 1905

12. Jorge BASADRE GROHMANN (1903-1980) 1946

13. Carlos F. BASADRe StEVEnSON (1857- ?) 1918?

14. Víctor Andrés Belaunde DieZ-CANSECo (1883-1966) 1908

15. Enrique Benites SACIO (1834-1917) 1905

16. Duccio Bonavia Berber (n. 1935) 1995

17. Crnl. Manuel C. Bonilla Castro (1873-1954) 1946?

18. Juan C. BRomley Seminario (1894-1968) 1955

19. José Luis Bustamante y Rivero (1894-1989) 1968

20. José Antonio del Busto DuthuRburu (n. 1932) 1967

21. Marco Aurelio Cabero Vega (1854-1934) 1905

22. Carlos Alfredo CAmprubí AlCÁZAR (1914-1970) 1960

23. Eduardo Percy CAYO CóRdova (n. 1937) 1980

24. Luis Jaime CisNeros VizQUerRa (n. 1921) 1995

25. Mariano Hilario CORNEJo CENTENO (1866-1942) 1905

26. José Gabriel Cosio Medina (1887-1960) 1945

27. Felipe Cossío del Pomar (1888-1981) 1981

28. Víctor L. CRiado y Tejada (1879-1960) 1921?

29. Rómulo Cúneo VIDAL (1856-1931) 1916?

30. Pedro Emilio Dancuart Galup (1847-1911) 1905

31. Gral. Carlos Dellepiane Alonso (1893-1946) 1936

32. Félix Denegri Luna (n. 1919) 1955

33. Carlos Deustua Pimentel (n. 1929) 1970 
34. Luis Antonio EGUIGUREN Escudero (1887-1967) 1946

35. Gral. Juan Norberto ElÉSPURU y Lasso de la Vega (1846-1923) 1905

36. José GÁLVEZ BARRENECHEA (1885-1957)

1919

37. Aníbal GÁlvez VALDERRAMa (1865-1922) 1905

38. Francisco GarCía-CALDERÓn Rey (1883-1953) 1949

39. Mons. Carlos García IrIGOYen (1857-1937) 1905

40. Ricardo García-Rosell OruÉ (1850-1924) 1905

41. Manuel Trinidad GonZÁLEZ de la Rosa (1841-1912)

42. Emilio GutiérReZ de Quintanilla y Flores (1858-1935) 1905

43. José Ramón de IdiÁQUEZ (1851-1905) 1905

44. José Augusto de IzCuE y GarCía (1872-1924) 1905

45. Federico KaufFManN Doig (n. 1928) 1995

46. Miguel Antonio de la Lama Dufoo (1839-1912) 1905

47. Rafael LARCo Hoyle (1901-1966) 1945

48. Carlos Larrabure y Correa (1876-1943) 1907

49. Eugenio LarRabURE y UnANUE (1844-1916) 1905

50. Juan Bautista de LAVAlLE y GarCía (1887-1970) 1911

51. Guillermo LoHMANN VilLena (n. 1915) 1955

52. Héctor LÓPEZ MARTíNeZ (n. 1935) 1982

53. Rafael LoREdo MENDívil (1892-1973) 1941

54. Miguel MATiCoRena Estrada (n. 1926) 1980

55. Víctor Manuel MaÚRTUA URIBE (1865-1937) 1905

56. Rosendo Melo Moreno (1847-1915) 1905

57. Oscar Miró-QueSADA de la GuerRa (1884-1981) 1920

58. Aurelio Miró-Quesada Sosa (n. 1907) 1947

59. Gral. Ernesto MontaGne MarkholZ (1885-1954) 1949

60. Manuel Moreyra y Paz SoldÁn (1894-1986) 1946

61. Jorge Clemente Muelle Rojas (1903-1974) 1955

62. Armando NiETo VéLEZ, S.J. (n. 1931) 1979

63. Manuel Jesús OBín y Charún (1841-1905) 1905

64. Teodorico Olaechea (1848-1905) 1905

65. Scarlett O'Phelan Godoy (n. 1951) 1995

66. Felipe de Osma y PARdo (1865-1924) 1921

67. César PACHeCo Vélez (1929-1989) 1970

68. José Simón PARDO y BARREDA (1864-1947) 1908

69. Pablo PATRÓN (1855-1910) 1905

70. Carlos Paz Soldán y Benavides (1844-1926) 1905

71. Franklin Pease García-YrigoYen (n. 1939) 1980

72. Jorge Polar VARGaS (1856-1932) 1908

73. José Toribio Polo ValenZuela (1841-1919) 1905

74. Raúl Porras BARRENECHEA (1897-1960) 1945

75. Ismael PORTAL ESPINOSA (1863-1934) - 
76. Javier Prado y Ugarteche (1871-1921)

1905

77. Mariano Ignacio Prado y Ugarteche (1870-1946)

1905

78. José Agustín de la Puente Candamo (n. 1922)

1960

79. José Agustín de la Puente Cortés (1838-1910)

1905

80. Pedro José RADA y GAMIO (1874-1938)

1921

81. José de la Riva Agüero y Osma (1885-1944)

1911

82. Emilio Romero PAdilla (1899-1993)

1947

83. Carlos Alberto Romero RAmírez (1863-1955)

1905

84. J. Salvador RoMERo SOTOMAYOR (1890-1951)

85. María Rostworowski de Diez Canseco (n. 1915)

1979

86. Evaristo SAN CRISTÓval PALOMINO (1894-1968)

1935

87. Luis Alberto SÁNCHEZ SÁNCHEZ (1900-1994)

1928

88. Fernando Silva-SANTISTEBAN BERnAl (n. 1929)

1995

89. Alberto TAURO del PINO (1914-1994)

1948

90. Julio César Tello Rojas (1880-1947)

91. Ella Dunbar Temple Aguilar (n. 1916)

1946

92. Gral. Oscar Nicolás ToRRES VELÁSQUEZ (1890-1975)

1947

93. Enrique Demetrio Tovar y RAMÍREz (1888-1947)

1995

94. Fernando de TrAZEGNIES GRANDA (n. 1935)

1980

95. Juan Manuel Ugarte ElÉSPURU (n. 1911)

1946

96. Pedro UGarteche Tizón (1902-1971)

1906

98. Luis UlloA Cisneros (1868-1936)

1963

99. Bolívar Ulloa Pasquette (n. 1909)

1965

101. Horacio H URTEAGA LÓPEZ (1873-1952)

102. Luis Eduardo VALCÁRCEL VizCARRA (1891-1987)

1932

103. José Manuel VALEGA VASALlo (1887-1961)

1945

104. Luis José Varela y Orbegoso (1878-1930)

1905

105. Rubén Vargas Ugarte, S.J. (1886-1975) 1931

106. Manuel Nemesio VARgas VALDIVIESO (1849-1921) 1905

107. José Miguel VéLEZ PiCASSO (1902-1971)

1968

108. Pedro Eduardo VILlar CóRdova (1901-1976) 1935

109. Carlos WiEsse Portocarrero (1859-1945) 1905

110. Crnl. Celso Nazario Zuleta y Heredia (1849-1924) 1905 